\title{
NOTE ON A THEOREM OF GELFAND AND ŠILOV
}

\section{FULTON KOEHLER}

The purpose of this note is to give a simplified proof of a theorem of Gelfand and Silov in the theory of normed, commutative rings.

Let $K$ be a complex Banach space which is also a commutative ring with unit element $e$, the norm being subject to the conditions $\|e\|=1$ and $\|x y\| \leqq\|x\|\|y\|$ for all $x$ and $y$ in $K$. Let $\mathcal{X}$ be the space of maximal ideals of $K$. Then, for each element $x \in K$, and each maximal ideal $M \in \mathscr{X}$, there is a unique complex number $x(M)$ defined by

$$
x \equiv x(M) e(\bmod M)
$$

and having the following properties:

$$
\begin{aligned}
e(M) & =1, \\
(x+y)(M) & =x(M)+y(M), \\
(x y)(M) & =x(M) y(M), \\
(\alpha x)(M) & =\alpha x(M), \\
|x(M)| & \leqq\|x\|,
\end{aligned}
$$

for all $x$ and $y$ in $K$ and any complex number $\alpha[1] .1$

Gelfand [1] introduces a topology in $\mathcal{X}$ by defining a neighborhood $U$ of $M_{0}$ as follows:

$U=\left\{M ;\left|x_{i}(M)-x_{i}\left(M_{0}\right)\right|<\alpha_{i} ; x_{i} \in K ; \alpha_{i}>0 ; i=1,2, \cdots, k\right\} ;$

and he proves that in this topology, $\mathcal{X}$ is a compact Hausdorff space, and that this is the unique topology in which all functions $x(M)$, $x \in K$, are continuous and $\mathcal{X}$ is compact.

If the ring $K$ also has the property that, for every $x \in K$, there exists an $x^{*} \in K$ such that $x(M)$ and $x^{*}(M)$ are complex conjugates for all $M$, then the functions $x(M)$ are dense in the set of all continuous functions on $\mathcal{X}$. This result is proved by Gelfand and Silov [2] by a method depending on two other topologies in the space $\mathcal{X}$. We give here a simpler and more direct proof of this theorem, making use of only the one topology defined above.

Lemma. If $F_{1}$ and $F_{2}$ are any two disjoint closed sets in $\mathcal{X}$, and $0<\epsilon_{1}<1,0<\epsilon_{2}<1$, there exists an $x \in K$ such that

Received by the editors July $24,1950$.

${ }^{1}$ Numbers in brackets refer to the references at the end of the paper. 


$$
\begin{array}{rlrl}
0 \leqq x(M) & \leqq 1, & & M \in \mathcal{H}, \\
0 \leqq x(M) \leqq \epsilon_{1}, & & M \in F_{1}, \\
1-\epsilon_{2} & \leqq x(M) \leqq 1, & & M \in F_{2} .
\end{array}
$$

Proof. Let $M_{0} \in F_{1}$. Let $U=\left\{M ;\left|x_{i}(M)-x_{i}\left(M_{0}\right)\right|<\alpha ; i\right.$ $=1,2, \cdots k\}$ be a neighborhood of $M_{0}$ which does not intersect $F_{2}$. Let $x_{i}^{\prime}=x_{i}-x_{i}\left(M_{0}\right) e, y^{\prime}=\sum_{i=1}^{k} x_{i}^{\prime}\left(x_{i}^{\prime}\right)^{*}$. Then $y^{\prime}(M)$ is non-negative for all $M$, is zero for $M=M_{0}$, and has a positive lower bound $\alpha^{2}$ for $M \in F_{2}$. Let $y=y^{\prime} /\left\|y^{\prime}\right\|, 2 \delta=\alpha^{2} /\left\|y^{\prime}\right\| \leqq 1$; then

$$
\begin{array}{cc}
0 \leqq y(M) \leqq 1, & M \in \mathcal{X}, \\
y\left(M_{0}\right)=0, & \\
0<2 \delta \leqq y(M) \leqq 1, & M \in F_{2} .
\end{array}
$$

With each $M_{0} \in F_{1}$ we associate a $y$ with the properties just given and a neighborhood of $M_{0}: U=\{M ; y(M)<\delta\}$. From these neighborhoods we select a finite covering of $F_{1}$, denoted by $U_{1}, U_{2}, \cdots$, $U_{N}$, and we let the $y$ and $\delta$ associated with $U_{k}$ be denoted by $y_{k}$ and $\delta_{k}$. Now let $z_{k}=e-\left(e-y_{k}^{n}\right)^{m}$, where $n$ and $m$ are positive integers. We then have

$$
\begin{array}{lr}
0 \leqq z_{k}(M) \leqq 1, & M \in \mathcal{X}, \\
0 \leqq z_{k}(M)<1-\left(1-\delta_{k}^{n}\right)^{m}, & M \in U_{k}, \\
1 .-\left(1-2^{n} \delta_{k}^{n}\right)^{m} \leqq z_{k}(M) \leqq 1, & M \in F_{\mathbf{z}} .
\end{array}
$$

If, for each value of $n$, we now choose $m$ as the integer nearest $\left(2 / 3 \delta_{k}\right)^{n}$, then, as $n \rightarrow \infty, m \log \left(1-\delta_{k}^{n}\right) \rightarrow 0$ and $m\left|\log \left(1-2^{n} \delta_{k}^{n}\right)\right| \rightarrow \infty$; hence $\left(1-\delta_{\boldsymbol{k}}^{n}\right)^{m} \rightarrow 1$ and $\left(1-2^{n} \delta_{\boldsymbol{k}}^{n}\right)^{m} \rightarrow 0$. This follows from the inequality $h<|\log (1-h)|<2 h$ for $0<h \leqq 1 / 2$. We can therefore choose $n$ so large that

$$
\begin{array}{cc}
0 \leqq z_{k}(M)<\epsilon_{1}, & M \in U_{k}, \\
\left(1-\epsilon_{2}\right)^{1 / N} \leqq z_{k}(M) \leqq 1, & M \in F_{2} .
\end{array}
$$

The function $x=z_{1} z_{2} \cdots z_{N}$ will then satisfy the conditions of the lemma.

ThEOREM. For any complex-valued function $f(M)$, continuous on $\mathcal{H}$, and any $\epsilon>0$, there exists an $x \in K$ such that $|f(M)-x(M)|<\epsilon$ for all $M$.

Proof. We first prove the theorem for a real-valued continuous function $f(M)$. Let $K_{R}$ be the set $\{x\}$ where $x \in K$ and $x(M)$ is realvalued, and let 


$$
a=\inf _{x \in K_{R}} \sup _{M \in \mathcal{X}}|f(M)-x(M)| .
$$

Let us assume $a>0$. Then there exists an $x_{0} \in K_{R}$ such that

$$
\sup _{M \in \mathcal{X}}\left|f(M)-x_{v}(M)\right|<1.1 a \text {. }
$$

Let $g(M)=f(M)-x_{0}(M)$, and let

$$
\begin{aligned}
F_{1} & =\{M ; g(M) \geqq .5 a\}, \\
G & =\{M ;|g(M)|<.5 a\}, \\
F_{2} & =\{M ; g(M) \leqq-.5 a\} .
\end{aligned}
$$

By the preceding lemma we can find $x_{1} \in K_{R}, x_{2} \in K_{R}$ such that

$$
\begin{array}{rlrlrl}
0 & \leqq x_{1}(M) \leqq .4 a, & 0 \leqq x_{2}(M) \leqq .4 a, & M \in \mathcal{X}, \\
0 \leqq x_{1}(M) & \leqq .1 a, & .3 a & \leqq x_{2}(M) \leqq .4 a, & M \in F_{2}, \\
.3 a & \leqq x_{1}(M) \leqq .4 a, & 0 \leqq x_{2}(M) \leqq .1 a, & M \in F_{1} .
\end{array}
$$

If $x=x_{0}+x_{1}-x_{2}$, then $x \in K_{R}$ and

$$
|f(M)-x(M)|<.9 a, \quad \text { for all } M \in \mathcal{X} \text {. }
$$

This is a contradiction; hence $a=0$ for any real-valued continuous $f(M)$.

If $f(M)$ is complex-valued, we can apply the result just proved to the real and imaginary parts of $f(M)$ separately.

\section{REFERENCES}

1. I. Gelfand, Normierte Ringe, Rec. Math. (Mat. Sbornik) N.S. vol. 9 (1941) pp. 3-24.

2. I. Gelfand and G. Silov, Ueber verschiedene Methoden der Einfuehrung der Topologie in die Menge der maximalen Ideale eines normierten Ringes, Rec. Math. (Mat. Sbornik) N.S. vol. 9 (1941) pp. 25-39.

The University of MinNesota 